\title{
The Effect of Gemfibrozil, Niacin and Cholestyramine Combination Therapy on Metabolic Syndrome in the Armed Forces Regression Study
}

\author{
Richard A. Krasuski, MD, Ganesh P. Devendra, BA, George Cater, BS, MS \\ and Edwin J. Whitney, MD
}

\begin{abstract}
Introduction: Metabolic syndrome is a powerful predictor of cardiovascular events independent of overt diabetes. Dietary restriction and weight loss modify metabolic syndrome components. This study addresses whether combination pharmacologic therapy focused on dyslipidemia provides additional benefit. Methods: This study examines the effect of 1 year of gemfibrozil, niacin and cholestyramine therapy on a baseline of aggressive dietary and lifestyle intervention in 143 clinically stable, nondiabetic patients with coronary disease, randomized into a double-blind, placebo-controlled trial. Results: Cohort characteristics included age $63 \pm 7$ years, $92 \%$ men, $43 \%$ with previous myocardial infarction, systolic blood pressure $139 \pm 17 \mathrm{~mm}$ $\mathrm{Hg}$, triglycerides $168 \pm 81 \mathrm{mg} / \mathrm{dL}$ and high-density lipoprotein cholesterol $34 \pm 6 \mathrm{mg} / \mathrm{dL}$. The mean number of metabolic syndrome components decreased from $2.2 \pm 0.9$ to $1.5 \pm 1.1, P<0.001$, and metabolic syndrome prevalence decreased from $38 \%$ to $18 \%(P<$ $0.001)$ for the entire cohort. In the lifestyle intervention and placebo group, the mean number of metabolic syndrome components decreased from $2.2 \pm 0.9$ to $1.9 \pm 1.1(P=0.01)$, and prevalence of metabolic syndrome decreased from $44 \%$ to $30 \%(P=0.15)$. A far more marked change was observed with lifestyle intervention and pharmacologic therapy: abnormal metabolic components decreased from $2.2 \pm 0.9$ to $1.0 \pm 1.0(P<0.001)$, and prevalence of metabolic syndrome decreased from $32 \%$ to $6 \%(P<0.001)$. Conclusions: The combination of gemfibrozil, niacin and cholestyramine has profound, beneficial effects on the components of metabolic syndrome. These benefits are additive to those seen with aggressive diet and lifestyle modification.
\end{abstract}

Key Indexing Terms: Metabolic syndrome; Diabetes; HDL; Exercise. [Am J Med Sci 2011;341(5):378-382.]

B cause of high calorie intake and a progressive reduction in physical activity, Americans have suffered from a $100 \%$ increase in the prevalence of obesity over the past 2 decades. ${ }^{1}$ More than $40 \%$ of Americans now perform no significant leisure-time physical activity, and more than $60 \%$ of our population is either obese or overweight. ${ }^{2}$ Gains in body weight are associated with greater degrees of insulin resistance ${ }^{3}$ and result in the development of dyslipidemia, hypertension and diabetes mellitus. ${ }^{4,5}$ The constellation of these findings has been coined the metabolic syndrome. The presence of metabolic syndrome is associated with increased cardiovascular morbidity and mortality and results in up to a 3-fold increase in

From the Division of Clinical Cardiology (RAK, GPD, GC), Department of CV Medicine, The Cleveland Clinic, Cleveland, Ohio; and Heart and Vascular Institute of San Antonio (EJW), San Antonio, Texas.

Submitted May 20, 2010; accepted in revised form August 13, 2010.

This study was supported by an unrestricted grant from Pfizer Pharmaceuticals.

Correspondence: Richard A. Krasuski, MD, Department of Cardiovascular Medicine, Desk F15, Cleveland Clinic Foundation, 9500 Euclid Avenue, Cleveland, OH 44195 (E-mail: krasusr@ccf.org). coronary artery disease and stroke. ${ }^{6}$ Although the presence of diabetes has been previously identified as a coronary disease equivalent, ${ }^{7}$ there is growing evidence that insulin resistance may itself be the culprit in accelerating atherosclerosis. ${ }^{8}$

Treatment for patients with metabolic syndrome has slowly been evolving. At the heart of risk modification is the recommendation for a healthy diet with a reduction in caloric intake appropriate for sustained weight loss. ${ }^{9,10}$ Patients should also be encouraged to participate in regular physical activity. ${ }^{11}$ Unfortunately, despite the proven efficacy of both diet and exercise intervention in treating metabolic syndrome, ${ }^{12,13}$ compliance with lifestyle changes remains a significant obstacle. ${ }^{14}$ Certainly, the presence of these hurdles would suggest a more expansive role for pharmaceutical intervention in metabolic syndrome.

The Armed Forces Regression Study was a randomized, double-blind, placebo-controlled trial of gemfibrozil, niacin and cholestyramine combination therapy on a baseline of aggressive dietary and lifestyle intervention. ${ }^{15}$ This substudy was designed to examine the effect of 1 year of combination drug therapy on the prevalence of metabolic syndrome in a population of nondiabetic patients with angiographic evidence of coronary artery disease. The beneficial effect of 30 months of combination therapy, including regression of angiographic stenosis and reduction in clinical events, has previously been reported for the entire cohort. ${ }^{15}$

\section{METHODS}

Men and women younger than 76 years with suspected stable coronary artery disease and a fasting lipid panel demonstrating low-density lipoprotein cholesterol (LDL-c) levels $\leq 160 \mathrm{mg} / \mathrm{dL}$ and high-density lipoprotein cholesterol (HDL-c) levels $<40 \mathrm{mg} / \mathrm{dL}$ were recruited after documented adherence to the American Heart Association (AHA) Step II diet for at least 6 months. The exclusions have previously been published $^{15}$ and included a major vascular event (myocardial infarction, cerebrovascular accident, coronary artery bypass grafting or other coronary catheter-based intervention) within 6 months, a history of congestive heart failure (other than in the setting of a myocardial infarction) or a left ventricular ejection fraction $<40 \%$ by ventriculography.

Participants were informed of the complete details of the study, and they enrolled voluntarily. Each patient signed an "Informed Consent Statement" that was reviewed and approved by the institutional review board at Wilford Hall Air Force Medical Center in San Antonio, Texas. Patient selection has been previously described in detail. ${ }^{15}$

After inclusion and exclusion criteria were met, a 6- to 8-month run-in period was performed to ensure that patients were capable of adhering to the prescribed diet. During this phase, all patients received dietary counseling from a study dietitian and exercise guidance from an exercise physiologist 
They were enrolled in a smoking cessation program if necessary. All study participants also committed to attend a bimonthly food show for training in the AHA Step II diet with reinforcing presentations from the dietitian, exercise specialist and cardiologist discussing cardiac risk factor modification.

Each participant was then block randomized to 1 of 2 treatment groups: pharmacologic therapy with gemfibrozil, niacin and cholestyramine and conventional therapy (which at the time of study enrollment did not include statins). The study was double blinded and placebo controlled. All medications and placebos were prepared by the pharmaceutical company and were dispensed at 30-day intervals. The pharmacologic therapy group began gemfibrozil at a dose of $600 \mathrm{mg}$ twice a day, and short-acting niacin was added in the third month at a dose of $250 \mathrm{mg}$ per day and titrated up to $3000 \mathrm{mg}$ per day as tolerated. Cholestyramine was added in the sixth month, and the dose was titrated up to $16 \mathrm{~g}$ per day as tolerated. The conventional therapy group was maintained on the AHA Step II diet and applicable matching placebos for the duration of the investigation. Provisions were made to administer cholestyramine in an open-label fashion if LDL-c exceeded $160 \mathrm{mg} / \mathrm{dL}$ during the trial.

Participants visited a single clinic on a monthly basis for the duration of the study. At each monthly visit, vital signs and weight were measured, unused study drugs were returned and counted, changes in medications or medical status were reviewed, new supplies of study drugs were provided and fasting blood was sampled. Full lipid profiles (including measured LDL-c), liver enzymes and fasting blood glucose were obtained monthly for the first 6 months and then bimonthly for the remainder of the study. Metabolic syndrome parameters were defined as a body mass index $(\mathrm{BMI}) \geq 30$, triglycerides $\geq 150$ $\mathrm{mg} / \mathrm{dL}$, a HDL-C $<50 \mathrm{mg} / \mathrm{dL}$ for women and $<40 \mathrm{mg} / \mathrm{dL}$ for men, a systolic blood pressure $\geq 130 \mathrm{~mm} \mathrm{Hg}$, and a fasting blood sugar $\geq 110 \mathrm{mg} / \mathrm{dL}$.

Data were compiled and analyzed using a statistical software package (JMP 8.0, (C) SAS Institute, Cary, NC). Comparison of continuous variables was performed using a 2-sample $t$ test on mean percent changes from baseline to 50 weeks of follow-up. Comparisons of dichotomous outcomes were performed using Fisher exact test or $\chi^{2}$ test where appropriate. Data are presented as mean \pm standard deviation for continuous variables and as a number (percentage) for dichotomous variables. A $P$ value less than or equal to 0.05 was considered statistically significant.

\section{RESULTS}

The baseline demographic information of the 143 patients randomized into the Armed Forces Regression Study is listed in Table 1. Seventy-one patients received the combination of gemfibrozil, niacin and/or cholestyramine and 72 received corresponding placebos. All but 2 patients in each group tolerated the goal dose of $600 \mathrm{mg}$ of gemfibrozil (or matching placebo) daily. The mean dose of niacin achieved in the treatment arm was $2.5 \pm 1.0 \mathrm{~g}$ daily, whereas the mean dose of cholestyramine was $8.4 \pm 6.2 \mathrm{~g}$ daily. Seventeen patients in the placebo arm and only 2 in the treatment arm received openlabel cholestyramine $(P<0.001)$. Patient characteristics were well balanced between the drug and placebo arms.

The lipid profiles, fasting blood sugars and blood pressures at the time of randomization are also listed in Table 1. Adherence to the AHA Step II diet was assessed by 3-day diet histories at study initiation, after 50 weeks of drug or placebo therapy and at the completion of the study. These demonstrate
TABLE 1. Baseline demographic data of patients randomized in the AFREGS trial

\begin{tabular}{lc}
\hline Demographics & $\begin{array}{c}\text { All patients } \\
(\mathbf{n}=\mathbf{1 4 3})\end{array}$ \\
\hline Age (yr; mean $\pm \mathrm{SD})$ & $63.2 \pm 7.1$ \\
Female (\%) & 7.7 \\
History of hypertension (\%) & 71.3 \\
History of angina (\%) & 65.0 \\
History of myocardial infarction (\%) & 43.4 \\
Previous smoker (\%) & 84.6 \\
Active smoker $(\%)$ & 8.4 \\
Weight (lbs; mean \pm SD) & $182.9 \pm 28.1$ \\
Body mass index (kg/m ${ }^{2} ;$ mean $\left.\pm \mathrm{SD}\right)$ & $26.5 \pm 3.7$ \\
Systolic blood pressure & $138.9 \pm 17.3$ \\
Diastolic blood pressure & $75.8 \pm 9.6$ \\
Fasting blood glucose & $81.8 \pm 13.6$ \\
Total cholesterol & $196.2 \pm 30.6$ \\
LDL cholesterol & $128.3 \pm 26.5$ \\
HDL cholesterol & $34.1 \pm 5.7$ \\
Triglycerides & $168.2 \pm 81$
\end{tabular}

AFREGS, Armed Forces Regression Study; SD, standard deviation; LDL, low-density lipoprotein; HDL, high-density lipoprotein.

remarkable compliance with the imposed dietary and caloric restrictions. The comparison of dietary parameters at study initiation and after 50 weeks of therapy is presented in Table 2.

Table 3 compares the change in bloodwork in the 2 groups observed after 50 weeks of therapy. Substantial reductions were seen in total cholesterol, LDL-C and triglycerides and an increase in HDL-C in the treatment arm compared with the placebo arm at 50 weeks. Both groups experienced a loss of weight and a decrease in BMI, although the magnitude was greater in the treatment arm. There was an increase in the fasting blood glucose in the treatment arm, although this did not translate to more diagnoses of diabetes using the widely accepted fasting glucose threshold of $126 \mathrm{mg} / \mathrm{dL}$. Reported adverse reactions to the medication regimen have been previously described ${ }^{15}$ and included skin rash, flushing and abdominal pain.

Figure 1 compares the distribution of abnormal metabolic parameters for the entire patient population at baseline and after 1 year. The prevalence of metabolic syndrome decreased from $37.9 \%$ to $18.0 \%$ during the course of the year

TABLE 2. Measures of compliance with American Heart Association Step II diet during the AFREGS trial

\begin{tabular}{llr}
\hline Measure & Time & All patients \\
\hline Calories & Baseline & $1545.4 \pm 430$ \\
& 50 weeks & $1555.9 \pm 453$ \\
Cholesterol (mg) & Baseline & $144.2 \pm 82.1$ \\
& 50 weeks & $155.5 \pm 81.4$ \\
Fat (\%) & Baseline & $22 \pm 8.1$ \\
& 50 weeks & $21 \pm 6.8$ \\
Saturated fat (\%) & Baseline & $4.3 \pm 2.2$ \\
& 50 weeks & $4 \pm 1.9$
\end{tabular}

${ }^{a}$ Mean \pm standard deviation.

AFREGS, Armed Forces Regression Study. 
TABLE 3. Percent changes in laboratory parameters in patients randomized in the AFREGS tria

\begin{tabular}{lccc}
\hline Percent change $^{\boldsymbol{a}}$ & Drug therapy & Placebo & $\boldsymbol{P}$ \\
\hline BMI & -4.8 & -0.8 & $<0.001$ \\
Systolic blood pressure $^{b}$ & -9.8 & -6.6 & 0.14 \\
Fasting glucose $^{b}$ & 17.6 & 8.1 & 0.006 \\
Total cholesterol & -16.7 & 2.8 & $<0.001$ \\
LDL cholesterol & -21.8 & 4.6 & $<0.001$ \\
HDL cholesterol & 37.9 & 2.0 & $<0.001$ \\
Triglycerides & -45.6 & 4.2 & $<0.001$ \\
\hline
\end{tabular}

${ }^{a} 100 \times($ value at 50 weeks - value at baseline)/(value at baseline).

${ }^{b}$ Zero patients had fasting glucose $\geq 126 \mathrm{mg} / \mathrm{dL}$ at baseline or at 1 year.

AFREGS, Armed Forces Regression Study; BMI, body mass index; LDL, low-density lipoprotein; HDL, high-density lipoprotein.

$(P<0.001)$. Figure 2 compares the distribution of abnormal metabolic parameters for both the treated and placebo groups at baseline and at 1 year, respectively. The prevalence of metabolic syndrome decreased from $43.5 \%$ to $30.4 \%$ in the placebo group $(P=0.15)$, whereas the prevalence of metabolic syndrome decreased from $32.4 \%$ to $5.7 \%$ in the treated group $(P<$ $0.001)$. There was not a significant difference in the prevalence of metabolic syndrome between the placebo and treatment groups at baseline $(P=0.22)$, but a significant difference was apparent $(P<0.01)$ at 1 year.

Table 4 shows the change in mean number of abnormal metabolic parameters during the observation period. In the placebo group, the mean number of metabolic syndrome components decreased from $2.2 \pm 0.9$ to $1.9 \pm 1.1(P=0.01)$. A far more marked change was observed in the treatment group with abnormal metabolic components decreasing from $2.2 \pm$
0.9 to $1.0 \pm 1.0(P<0.001)$ There was no significant difference in the number of abnormal metabolic parameters among patients assigned to placebo and treatment groups at baseline $(P=0.73)$, but a significant difference was noted $(P<$ $0.001)$ at 1 year.

\section{DISCUSSION}

In a population of patients already undergoing aggressive diet and exercise modifications to reduce cardiovascular risk factors, the addition of combination pharmacologic therapy significantly decreased the prevalence of metabolic syndrome after only a year of therapy. Although previous studies have indicated that weight reduction and regular exercise are the most important therapeutic interventions for the treatment of metabolic syndrome, ${ }^{16}$ to our knowledge, this study is the first to imply that drug therapy in combination with lifestyle modifications has profound, additional benefit on metabolic syndrome components.

In the analysis of this extremely compliant patient population, there was an overall reduction in the mean number of metabolic syndrome components from $2.2 \pm 0.8$ to $1.5 \pm 1.1$ $(P<0.001)$, with a corresponding decrease in metabolic syndrome prevalence from $37.9 \%$ to $18.0 \%(P<0.01)$ after 1 year of intervention. Those who received placebo on top of diet and exercise modification had a modest improvement in metabolic syndrome prevalence $(43.5 \%$ to $30.4 \%, P=0.15)$ and mean number of abnormal metabolic parameters $(2.2 \pm 0.9$ to $1.9 \pm 1.1, P=0.01)$. These results are consistent with previous reports that demonstrated the benefit of cardiac rehabilitation and exercise training on metabolic syndrome. ${ }^{17}$ Interestingly, those who received pharmacologic therapy on top of diet and exercise modification demonstrated a more robust improvement in prevalence of metabolic syndrome $(32.4 \%$ to $5.7 \%$, $P<0.001)$ and mean number of abnormal metabolic parameters $(2.2 \pm 0.9$ to $1.0 \pm 1.0, P<0.001)$.

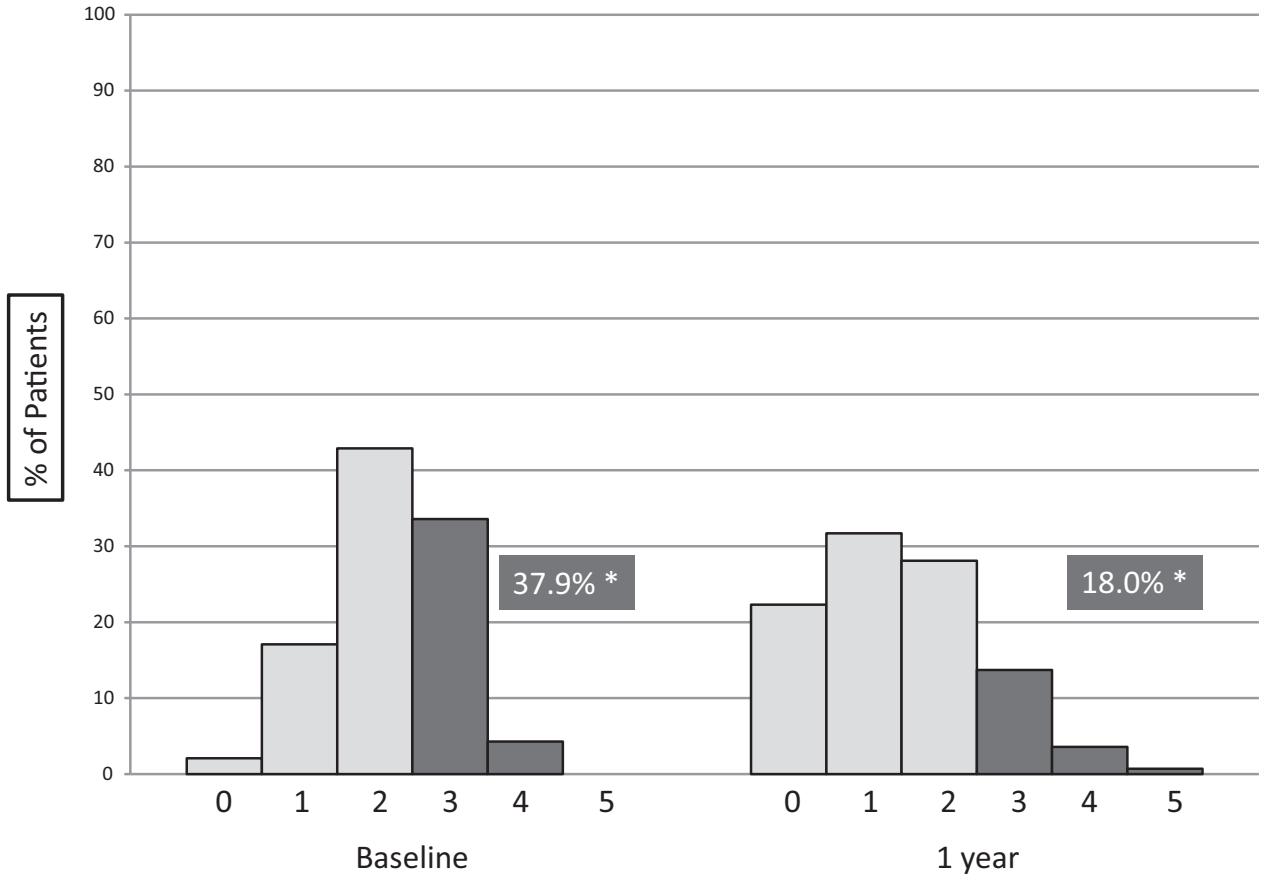

FIGURE 1. Distribution of abnormal metabolic parameters at baseline and at 1 year for all patients. Number of metabolic syndrome components decreased from $2.2 \pm 0.9$ to $1.5 \pm$ 1.1, $P<0.001$. *Prevalence of metabolic syndrome decreased from $37.9 \%$ to $18.0 \%, P<0.001$.

\# of Abnormal Metabolic Parameters 


\section{A Baseline Placebo}

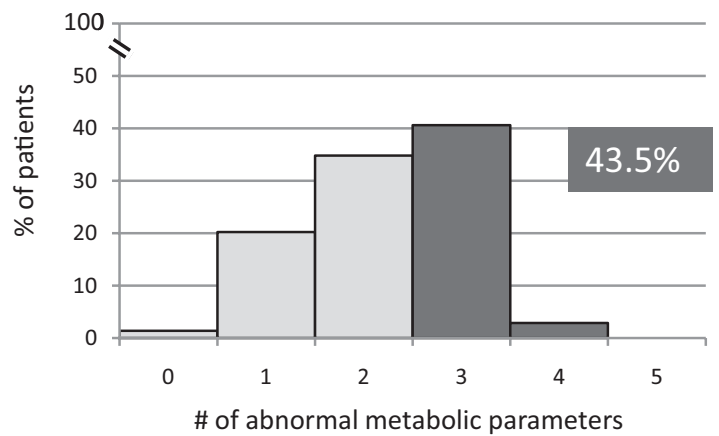

\section{Baseline Treated}

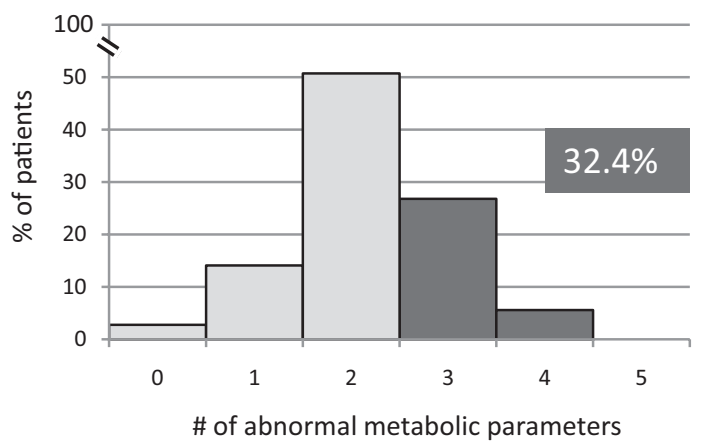

$\mathrm{P}=0.22$

\section{B 1 year Placebo}

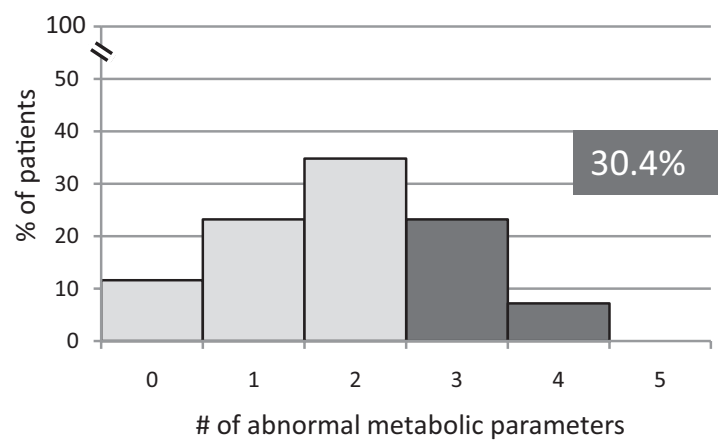

$\mathrm{P}=0.15$

D 1 year Treated

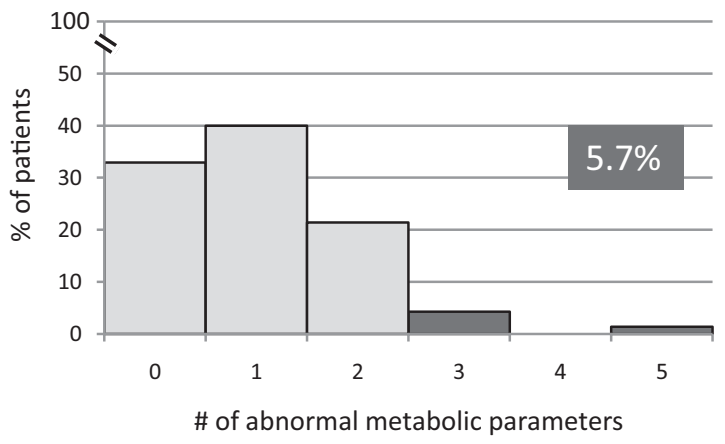

$\mathrm{P}<0.001$

FIGURE 2. Distribution of abnormal metabolic parameters in treated and untreated patients at baseline and after 1 year.

These results confirm the benefits of dietary modifications and regular exercise on factors such as BMI, glucose tolerance, hypertension and hyperlipidemia. Interestingly, however, significant differences were noted in many of these factors between the control and combination drug therapy groups. It would be expected that significant improvements in the lipid profile would be seen with pharmacologic therapy, because the therapeutic targets of gemfibrozil, niacin and cholestyramine lead to reductions in LDL-C and triglycerides and increases in HDL-C. The significant decrease in BMI in patients receiving drug therapy, however, also suggests other, less well-defined targets of these agents, which may lead to reductions in body weight and ultimately impact the other components of metabolic syndrome. It is important to note that these improvements occurred in the context of a significant increase in fasting blood glucose, undoubtedly the result of niacin. It has previously been suggested that niacin should be avoided in patients with insulin

TABLE 4. Mean number of abnormal metabolic parameters at baseline and at 1 year

\begin{tabular}{lccc}
\hline & Baseline & 1 Year & $\boldsymbol{P}$ \\
\hline Placebo & $2.2 \pm 0.9$ & $1.9 \pm 1.1$ & 0.01 \\
Treated & $2.2 \pm 0.9$ & $1.0 \pm 1.0$ & $<0.001$ \\
$P$ & 0.73 & $<0.001$ & \\
\hline
\end{tabular}

resistance for this reason, and insulin resistance seems to be the backbone of metabolic syndrome. Despite these adverse effects on glycemic control, no patients developed overt diabetes, and the other components of the metabolic syndrome were improved to the extent that metabolic syndrome prevalence was actually reduced. These results reflect those of other previous studies, including the Arterial Disease Multiple Intervention Trial, ${ }^{18}$ and suggest safety of niacin-based regimens in such patients.

Furthermore, although the prevalence of metabolic syndrome in both groups of patients was similar at baseline, the pharmacologic therapy group had a significant reduction in metabolic syndrome than the group that received diet and exercise alone. This demonstrates that adjunctive pharmacologic therapy targeted at optimizing lipid levels provides enhanced outcomes in the population of patients who have metabolic syndrome and have yet to progress to diabetes. With modifications of the current therapeutic approach, it may be possible to further reduce the incidence of the metabolic syndrome and thus reduce the risk for cardiovascular events. Our analysis is limited by the fact that none of these patients were treated with a statin drug, which would now be standard of care for a majority of patients in this cohort. The administration of a statin would of course contraindicate the inclusion of gemfibrozil in the regimen.

In summary, these data support the current guidelines of lifestyle changes to combat metabolic syndrome and suggest 
that they can successfully accomplish this goal. However, the addition of a therapeutic regimen using gemfibrozil, niacin and cholestyramine can further reduce metabolic syndrome in these patients. The mechanism by which this occurs seems to be through the optimization of the lipid profile and reduction in BMI. Although glucose levels may increase, they do not seem to result in the development of diabetes. Our study size was small and the population was predominantly men, making the applicability to female patients less clear. Our analysis is strengthened, however, by a compliant patient population, allowing for the true evaluation of the interventions studied. The fact that the study was conducted in the pre-statin era allows insight into a nontraditional approach and should stimulate consideration of a head-to-head comparison with statin therapy in this high cardiovascular risk patient population.

\section{REFERENCES}

1. Khan LK, Sobush K, Keener D, et al. Recommended community strategies and measurements to prevent obesity in the United States. MMWR Recomm Rep 2009;58:1-26.

2. Ogden CL, Carroll MD, Curtin LR, et al. Prevalence of overweight and obesity in the United States, 1999-2004. JAMA 2006;295:1549-55.

3. Pereira MA, Kartashov AI, Ebbeling CB, et al. Fast-food habits, weight gain, and insulin resistance (the CARDIA study): 15-year prospective analysis. Lancet 2005;365:36-42.

4. Colditz GA, Willett WC, Rotnitzky A, et al. Weight gain as a risk factor for clinical diabetes mellitus in women. Ann Intern Med 1995; 122:481-6.

5. Lavie CJ, Milani RV, Ventura HO. Obesity and cardiovascular disease: risk factor, paradox, and impact of weight loss. J Am Coll Cardiol 2009;53:1925-32.

6. Isomaa B, Almgren P, Tuomi T, et al. Cardiovascular morbidity and mortality associated with the metabolic syndrome. Diabetes Care 2001; 24:683-9.

7. Juutilainen A, Lehto S, Ronnemaa T, et al. Type 2 diabetes as a "coronary heart disease equivalent": an 18-year prospective populationbased study in Finnish subjects. Diabetes Care 2005;28:2901-7.
8. Lempiainen $\mathbf{P}$, Mykkanen $\mathbf{L}$, Pyorala $\mathbf{K}$, et al. Insulin resistance syndrome predicts coronary heart disease events in elderly nondiabetic men. Circulation 1999;100:123-8.

9. Wagh A, Stone NJ. Treatment of metabolic syndrome. Expert Rev Cardiovasc Ther 2004;2:213-28

10. Pasanisi F, Contaldo F, de Simone G, et al. Benefits of sustained moderate weight loss in obesity. Nutr Metab Cardiovasc Dis 2001;11: 401-6.

11. Pacholczyk M, Ferenc T, Kowalski J. [Metabolic syndrome. Part III: its prevention and therapeutic management]. Postepy Hig Med Dosw (Online) 2008;62:559-70.

12. Singh RB, Singh NK, Rastogi SS, et al. Effects of diet and lifestyle changes on atherosclerotic risk factors after 24 weeks on the Indian Diet Heart Study. Am J Cardiol 1993;71:1283-8.

13. Anderssen SA, Hjermann I, Urdal P, et al. Improved carbohydrate metabolism after physical training and dietary intervention in individuals with the "atherothrombogenic syndrome." Oslo Diet and Exercise Study (ODES). A randomized trial. J Intern Med 1996;240:203-9.

14. Fappa E, Yannakoulia M, Pitsavos C, et al. Lifestyle intervention in the management of metabolic syndrome: could we improve adherence issues? Nutrition 2008;24:286-91.

15. Whitney EJ, Krasuski RA, Personius BE, et al. A randomized trial of a strategy for increasing high-density lipoprotein cholesterol levels: effects on progression of coronary heart disease and clinical events. Ann Intern Med 2005;142:95-104.

16. Jiamsripong $\mathbf{P}$, Mookadam M, Alharthi MS, et al. The metabolic syndrome and cardiovascular disease: part 2. Prev Cardiol 2008;11: 223-9.

17. Milani RV, Lavie CJ. Prevalence and profile of metabolic syndrome in patients following acute coronary events and effects of therapeutic lifestyle change with cardiac rehabilitation. Am J Cardiol 2003;92: $50-4$.

18. Chesney CM, Elam MB, Herd JA, et al. Effect of niacin, warfarin, and antioxidant therapy on coagulation parameters in patients with peripheral arterial disease in the Arterial Disease Multiple Intervention Trial (ADMIT). Am Heart J 2000;140:631-6. 